\title{
Digital Image Compression using Hybrid Transform with Kekre Transform and Other Orthogonal Transforms
}

\author{
H.B. Kekre ${ }^{1}$, Tanuja Sarode ${ }^{2}$, Prachi Natu ${ }^{3}$ \\ ${ }^{1}$ (Department of Computer Engineering, MPSTME/ NMIMS University, India) \\ ${ }^{2}$ (Department of Computer Engineering, ThadomalShahaniEngg. College/Mumbai University, India) \\ ${ }^{3}$ (Department of Computer Engineering, MPSTME/ NMIMS University, India)
}

\begin{abstract}
This paper presents image compression technique using hybrid transform. Concept of hybrid wavelet transform can be extended to generate hybrid transform. In hybrid wavelet transform first few rows represent global features of an image and remaining rows represent local features of an image. In Hybrid wavelet matrix rows contributing to global characteristics can be varied. In the limiting case by taking kronecker product of to orthogonal component transforms, hybrid transform is generated where all rows of transform matrix represent global features and no local features are present. This hybrid transform matrix is then applied on color image. High frequency contents of transformed image are eliminated and only low frequency contents are retained to get compressed image. RMSE is calculated at different compression ratios to check the performance of hybrid transforms. Various orthogonal transforms like DCT,Walsh, Slant, Hartley, Real-DFT and DST are combined with Kekre transform to generate hybrid transforms. DKT-DCT gives better image quality and lower RMSE than other pairs formed with DKT. Component size 32-8 i.e.32x32(Kekre Transform) and $8 \times 8$ (DCT) gives best results than other possible size combinations like 8-32,16-16 and 64-4.
\end{abstract}

Keywords: Compression Ratio, Hybrid Transform, Image compression, Kekre Transform, Real-DFT

\section{Introduction}

In today's multimedia applications, digital images are used on large scale. Storage and transmission of such images needs more memory and bandwidth. Also time required to transfer such large amount of information is more.This infeasibility can be avoided by compressing the images. In compression, only visible information is extracted and redundant information is eliminated. [1]. It results in less storage spaceand less time for transmission.Image compression falls in two classes: lossy and lossless. In lossy image compression some loss of clearness of an image is allowed as it is not detected by human eyes. Discrete Cosine transform (DCT) [2] is a popular transform used in image compression. While using DCT image is divided into blocks and then DCT is applied on blocked image. It introduces blocking artifacts in the compressed image.

Wavelet transform is a mathematical tool that divides the data into different frequency components. High energy compaction property is the key characteristic of wavelets. Wavelets also help to analyze local properties of an image. This feature makes them highly applicable in image compression. Nowadays wavelets are becoming popular for other applications like biometrics applications [3,4], CBIR [5],steganography [6], analysis of DNA, ECG etc. Various wavelet based compression schemes are available and implemented in literature [7]. Different wavelet based image coding schemes include lifting based wavelet transform[8],set partitioning in hierarchical trees(SPIHT)[9,10],spatial orientation tree wavelet(STW), Embedded zero tree wavelet(EZW), Wavelet difference reduction (WDR) and adaptively scanned wavelet difference reduction(ASWDR)[1].

\section{Related Work}

Various methods have been proposed by different researchers in the literature. In recent years focus is on wavelet based compression methods and hybrid compression techniques. Compression using column and row transform is proposed in [11] by Kekre et al. Column and row wavelet based image compression is presented in [12]by Kekre et al. which uses wavelet generation method proposed in [13]. Use of column transforms or column wavelet transforminstead of full transform or full wavelet transform proves to be useful in saving number of computations. Real Discrete Fourier Transform has been proposed in [14] by H.B.Kekre, TanujaSarode and PrachiNatu. It considers only real valued functions in Fourier transform and avoids complex functions.Hybrid compression technique using DCT and fractal image compression method has been proposed byRawat and Meher in[15]. DCT is applied on 8x8 blocked images and then DCT coefficients of each block are quantized. Zigzag scanning is used to extract nonzero coefficients.Further Fractal image compression method is used and then the image will be encoded using Huffman coding.In [16] color image compression using DCT, VQ based coding and a new method that combines DCT and wavelet transform is used. Fractal image coding using optimization techniques like genetic algorithm, ant colony optimization and particle swarm optimization is 
given in [17]. An attempt to reduce the computational cost is done by using these optimization techniques. In [18] effective run length coder has been implemented. Their algorithm works on quantized coefficients of DCT.DCT based fractal image compression and wavelet based fractal image compression is proposed in[19]. In[20] wavelet families like Daubechies, Biorthogonal, Coeflit and Symlet have been examined. Combination of wavelet image compression with SPIHT and fractal image compression has been presented in [21].

\section{Proposed Method}

In this paper hybrid transform is generated from two orthogonal transforms and applied on the image. In [22] generation of hybrid wavelet transform from two orthogonal transforms has been proposed.Kekre transform [23] is used as base transform to generate hybrid transform. Consider two orthogonal transforms A and $\mathrm{B}$ having size $\mathrm{MxM}$ and $\mathrm{NxN}$ respectively. To generate hybrid wavelet of $\mathrm{A}$ and $\mathrm{B}$, first ' $\mathrm{m}$ ' rows of resultant matrix is calculatedby repeating each column of a' $\mathrm{N}$ ' times and multiplying it with each element of first row of $B$. These ' $M$ ' rows represent global characteristics in hybrid wavelet transform. Remaining rows are obtained by translating the rows of matrix B from second row onwards. These rows contribute local features of an image. Generated hybrid wavelet transform is as follows:

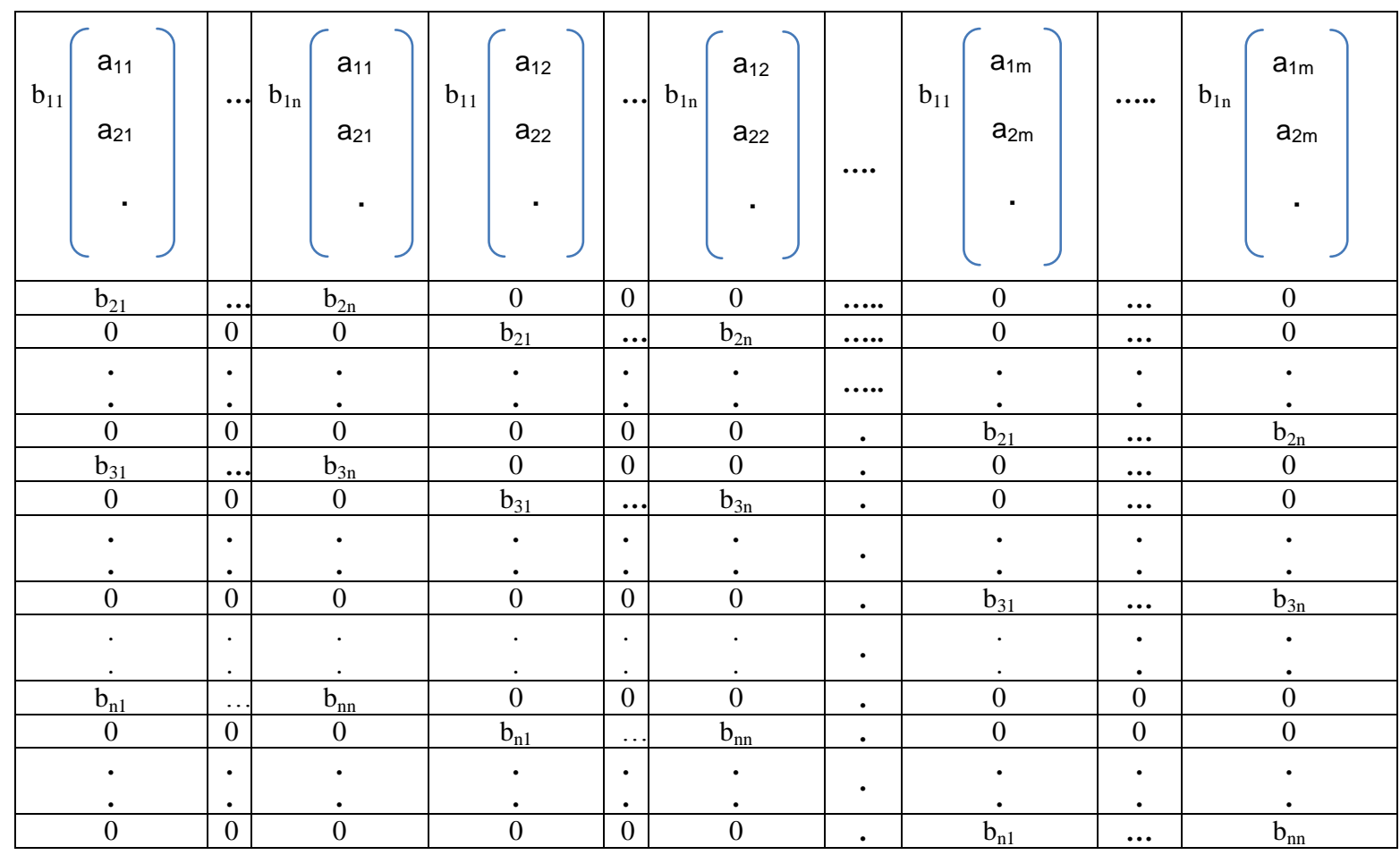

To increase contribution of global features, hybrid wavelet formation is done differently as in [24]. First $\mathrm{N}$ rows of $\mathrm{T}_{\mathrm{AB}}$ are formed by repeating each column of ' $\mathrm{A}$ ' ' $\mathrm{N}$ ' times and multiplying each column by each element of first row of $B$. Next $M$ rows are formed by repeating each column of ' $A$ ' $N$ times and multiplying each column by each element in second row of B.Remaining (N-2)M rows are obtained by performing shift and rotate operation on third row onwards of Matrix ' $\mathrm{B}$ ' by appending zeroes to it. As contribution of global characteristics increases, contribution of local features decreases.Above hybrid wavelet transform matrix changes to

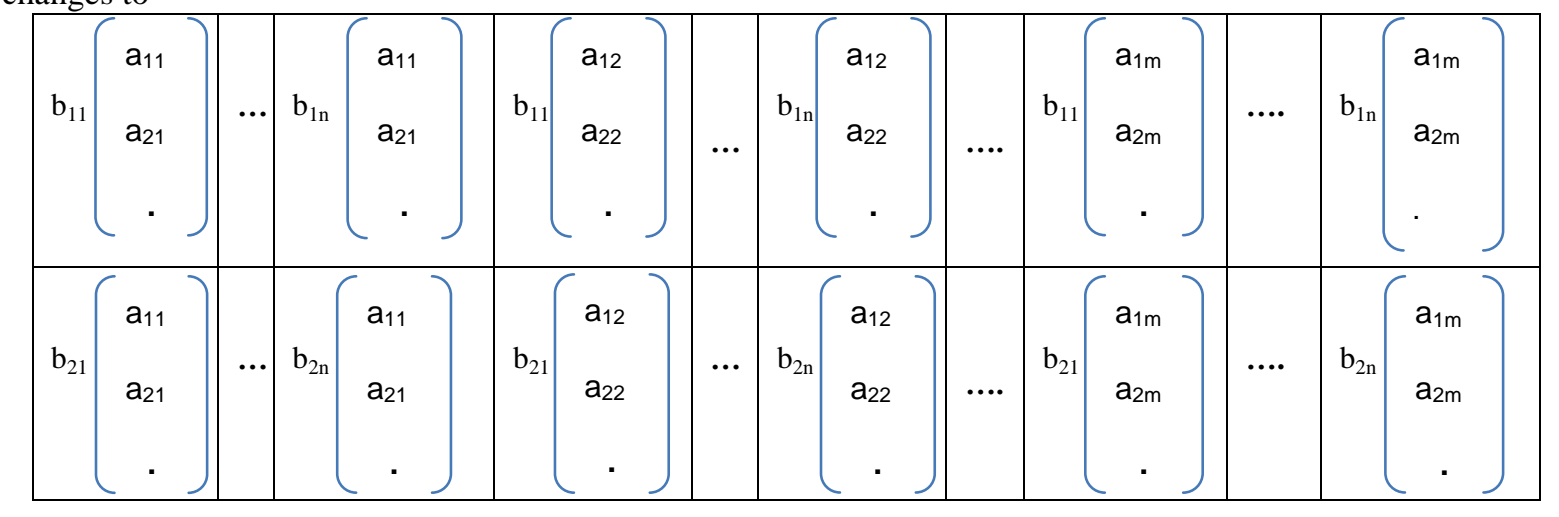




\begin{tabular}{|c|c|c|c|c|c|c|c|c|c|}
\hline $\mathrm{b}_{31}$ & . & $b_{3 n}$ & 0 & 0 & 0 & $\begin{array}{l}\cdots \\
\ldots .\end{array}$ & 0 & 0 & 0 \\
\hline 0 & 0 & 0 & $\mathrm{~b}_{31}$ & . & $b_{3 n}$ & $\begin{array}{l}\ldots \\
\ldots .\end{array}$ & 0 & 0 & 0 \\
\hline$\dot{.}$ & $\dot{.}$ & . & . & $\dot{.}$ & . & $\begin{array}{l}\ldots \\
\ldots .\end{array}$ & . & $\dot{.}$ & . \\
\hline 0 & 0 & 0 & 0 & 0 & 0 & $\begin{array}{l}\ldots \\
\ldots .\end{array}$ & $\mathrm{b}_{31}$ & . & $b_{3 n}$ \\
\hline • & . & • & • & . & . & $\begin{array}{l}\cdots \\
\ldots .\end{array}$ & . & $\dot{.}$ & . \\
\hline $\mathrm{b}_{\mathrm{n} 1}$ & . & $\mathrm{b}_{\mathrm{nn}}$ & 0 & 0 & 0 & $\ldots$ & 0 & 0 & 0 \\
\hline 0 & 0 & 0 & $\mathrm{~b}_{\mathrm{n} 1}$ & . & $\mathrm{b}_{\mathrm{nn}}$ & $\begin{array}{l}\cdots \\
\cdots \\
\cdots\end{array}$ & 0 & 0 & 0 \\
\hline . & . & . & . & . & . & - & . & . & . \\
\hline 0 & 0 & 0 & 0 & 0 & 0 & 0 & $b_{n 1}$ & . & $\mathrm{b}_{\mathrm{nn}}$ \\
\hline
\end{tabular}

Hybrid transform is the limiting case of hybrid wavelet transform generated above in which only global characteristics are focused and local features are not considered.It is generated as $\mathrm{A} \otimes \mathrm{B}$. Hybrid transform matrix is given below:

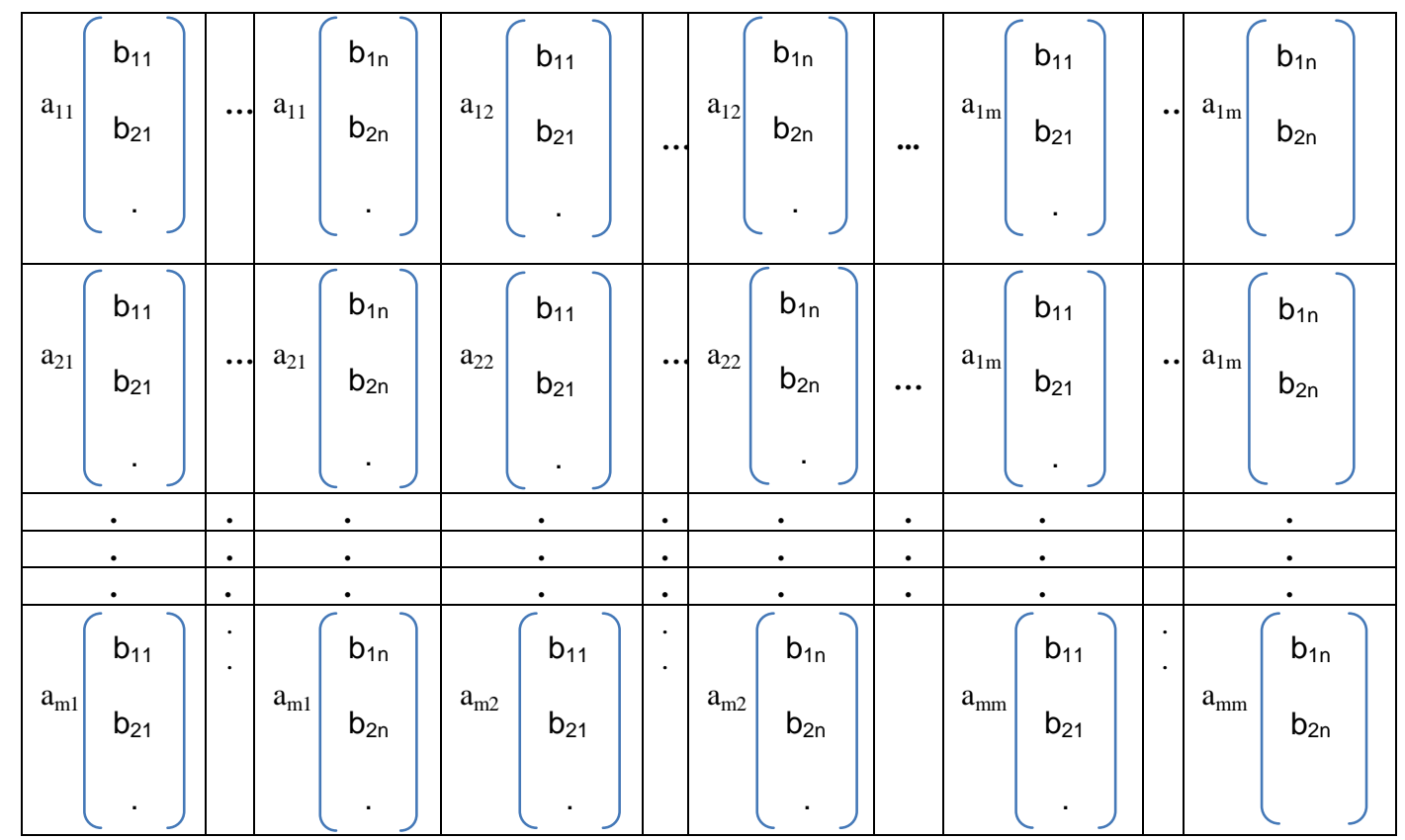

Above hybrid transform matrix is applied on R, G, and B plane of image separately. To compress the image low frequency contents of image are retained and high frequency contents are made zero. Compression ratio is varied from 2 to 32. In each case Root Mean Square Error between original image and compressed image is calculated.

\section{Experiments and Results}

Proposed method is experimented on 20 different images using Matlab 7 and AMD dual core processor with 4 GB RAM.

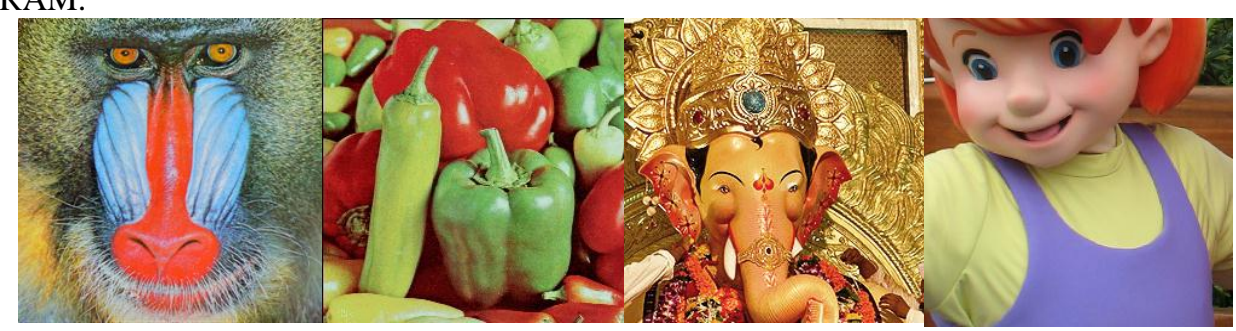




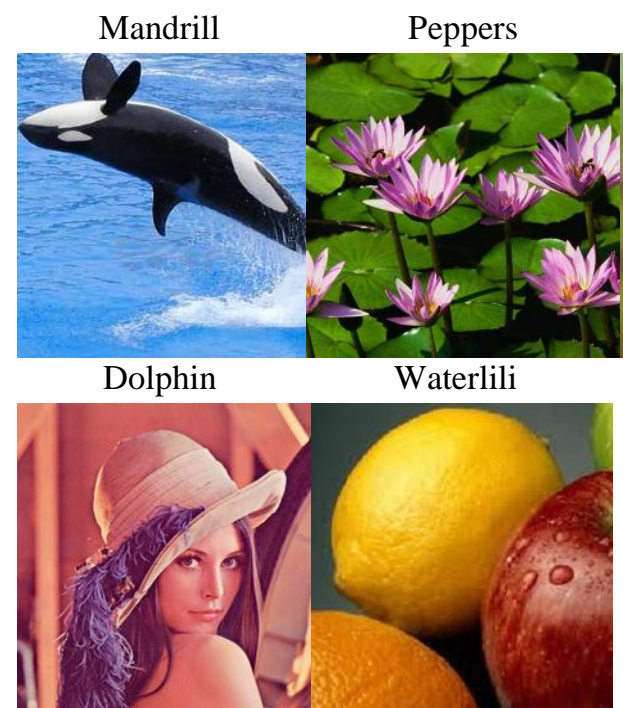

Lord Ganesha

Cartoon

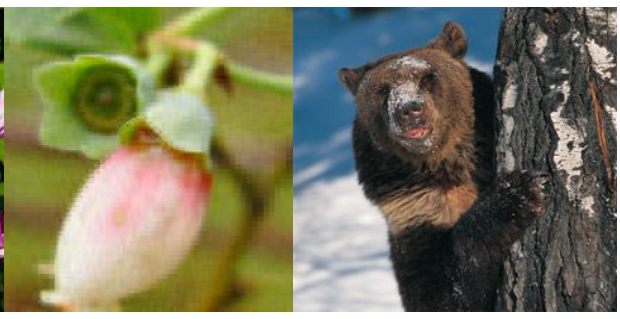

Lena Apple
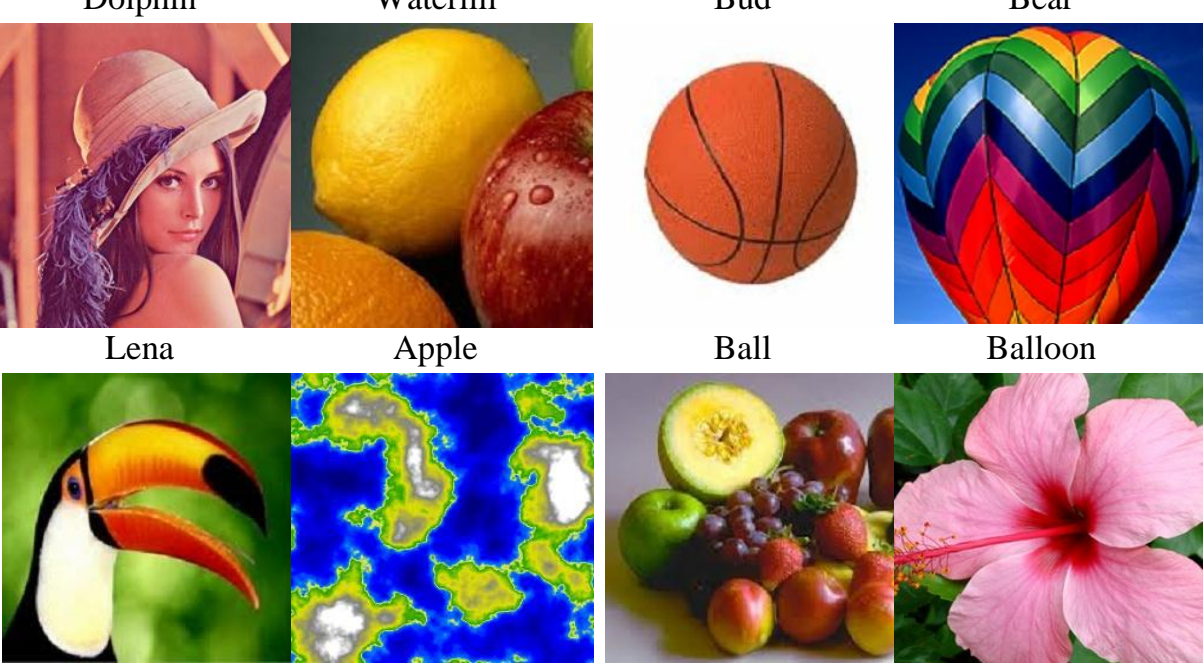

Ball

Balloon

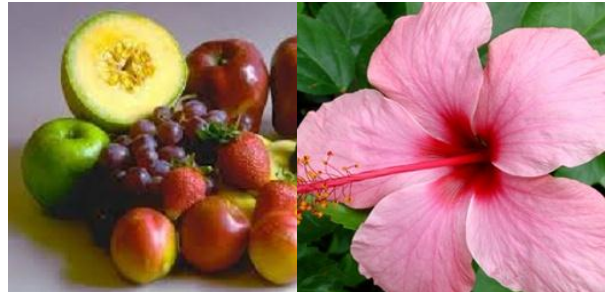

Bird

Colormap

Fruits

Hibiscus

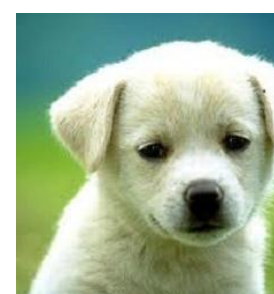

Puppy

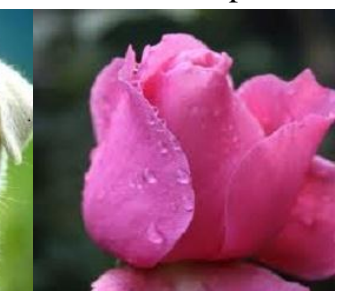

Rose

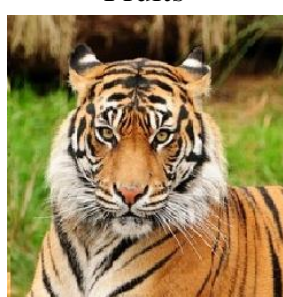

Tiger

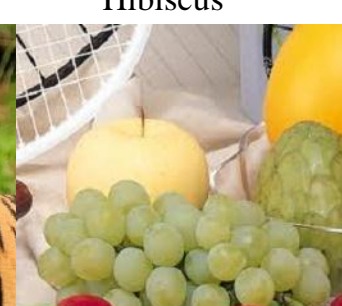

Grapes

Fig.1 Set of twenty test images of different classes used for experimental purpose

Initially Walsh transform is used as matrix 'A' and Kekretransform is used as matrix ' $\mathrm{B}$ '. Thus WalshDKT hybrid transform is generated and applied on the images. Performance is measured in terms of RMSE at different compression ratios extending up to 32 . Then hybrid transform is generated by reversing the matrix ' $\mathrm{A}$ ' and ' $\mathrm{B}$ '. i.e. by using DKT-Walsh hybrid transformsand again performance is measured. It is compared with the performance of Walsh-DKT hybrid transform. Similarly performance of DCT-DKT and DKT-DCT hybrid transform is compared. Resulting graphs related to these performances are shown below.

Fig. 2 compares performance of Walsh-DKT and DKT-Walsh hybrid transform. Various size combinations of component transforms are tried to select combination giving least RMSE. It has been observed that DKT-Walsh hybrid transform gives less error as compared to Walsh-DKT. Minimum RMSE at various compression ratios is plotted against compression ratio. Among four different size combinations 64-4size DKTWalsh gives lower value of error upto compression ratio 10. For higher compression ratios 16 and 32, DKTWalsh for size 32-8 gives gives lower RMSE. It is observed that as compression ratio increases RMSE increases. The lowest RMSEis 1.57 at compression ratio 2. It increases to 13.94 for compression ratio 32. 


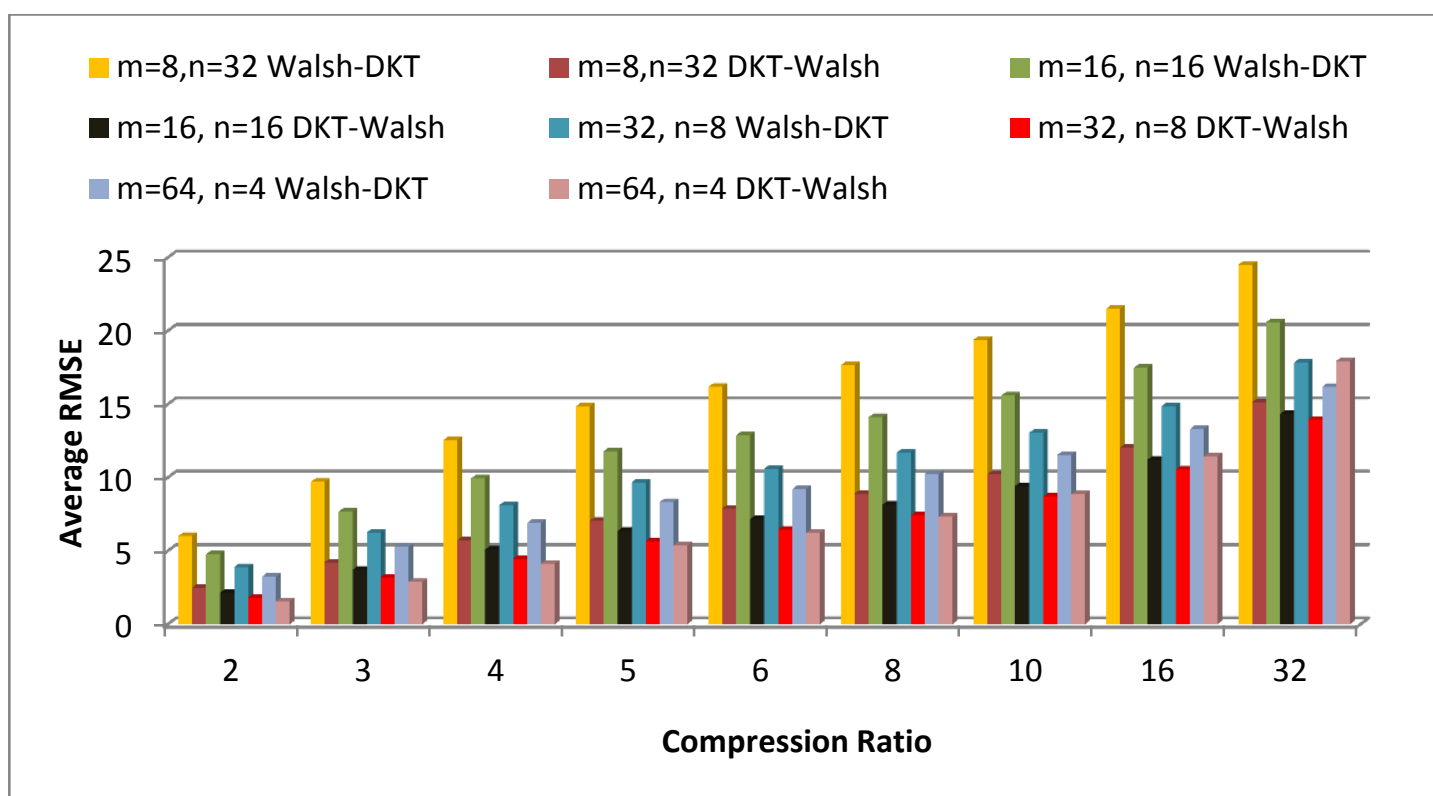

Fig.2Comparison of Avg. RMSE vs Compression Ratio exchanging 'A' and 'B' matrices as Walsh-DKT and DKT-Walsh Hybrid Transform

Fig. 3 compares results obtained by DCT-DKT and DKT-DCT hybrid transform using various sizes of component transforms.DKT-DCT gives less error than DCT-DKT for all combinations. Upto compression ratio 6, component size 32-8 and 64-4 of DKT-DCT gives nearly same error. Thereafter from compression ratio 8 to 16 DKT-DCT using component size 32-8 gives less error than size 64-4. At compression ratio 32, component size 16-16 of DKT-DCT gives better results than others. The lowest RMSE is 1.34 at compression ratio 2. It increases to 11.90 for compression ratio 32.Thus giving less error as compared to DKT-Walsh for all combinations.

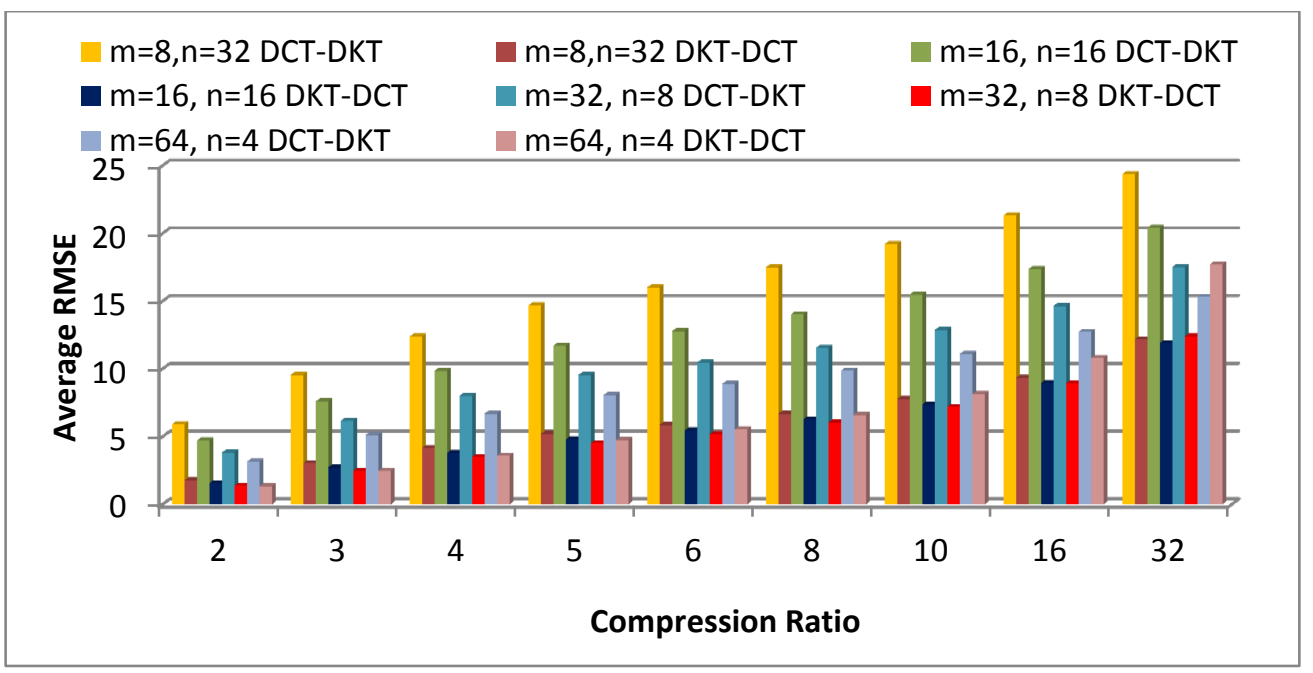

Fig.3Comparison of Avg. RMSE against Compression Ratio by exchanging the base transform and local transform as Walsh-DCT and DCT-Walsh Hybrid Wavelet Transform

As observed in above two cases DKT-DCT gives better results than DKT-Walsh.Now with Kekre transform other orthogonal transforms like Real-DFT, Hartley, Discrete Sine Transform (DST) and Slant transforms are used. Their performances are compared for different component sizes and are plotted in graphs below.

Fig. 4 compares results obtained by different transforms combined with Kekre transform. Kekre transform of size $32 \times 32$ and second component transform of size $8 \times 8$ is selected. Among six different hybrid transforms DKT-DCT gives lower RMSE. Acceptable image quality is obtained at compression ratio 32. Performance of DKT-DCT is closely followed by DKT-RealDFT combination. 


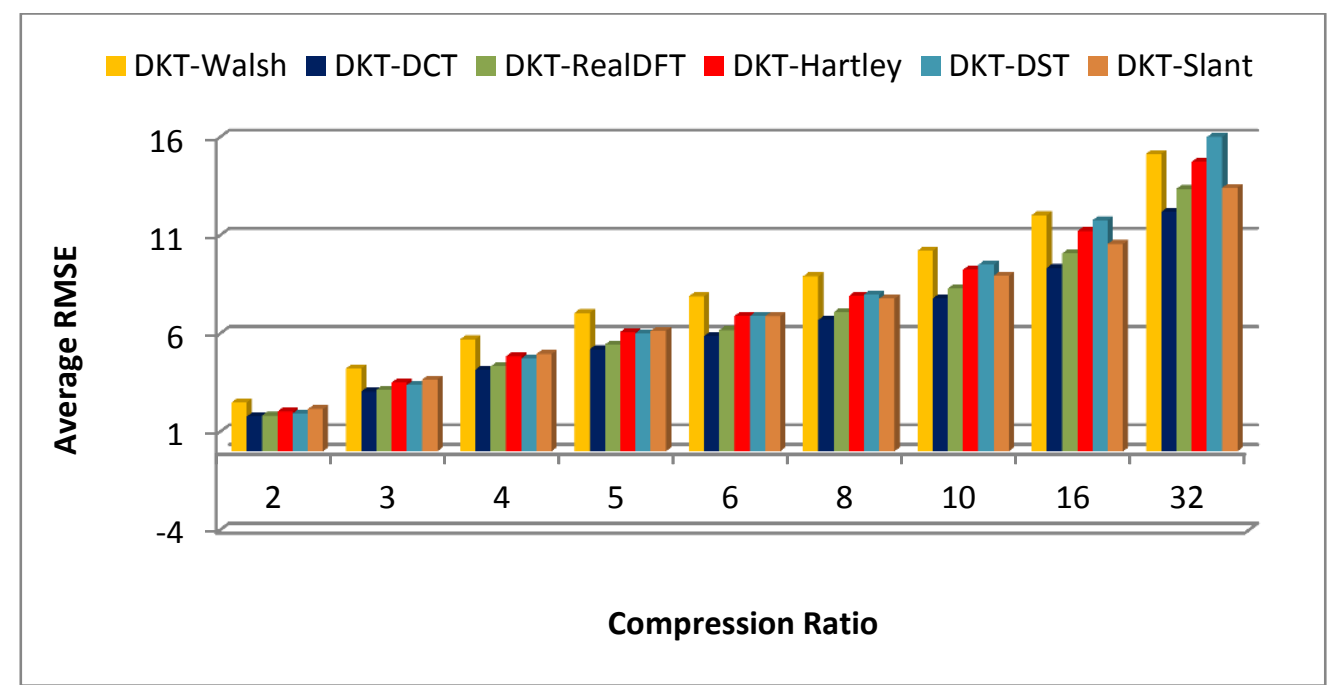

Fig.4Average RMSE against Compression Ratio for Kekre Hybrid Wavelet Transform where 8x8

KekreTransform acting as a base transform and different local component transforms of size $32 \times 32$

Fig. 5 compares performance of all hybrid transforms with both component transforms of size 16. At this component size also DKT-DCT shows superior performance amongst all. DKT-Real-DFT follows DKTDCT upto compression ratio 8. For higher compression ratios from 10 to 32 DKT-Slant gives second best performance.

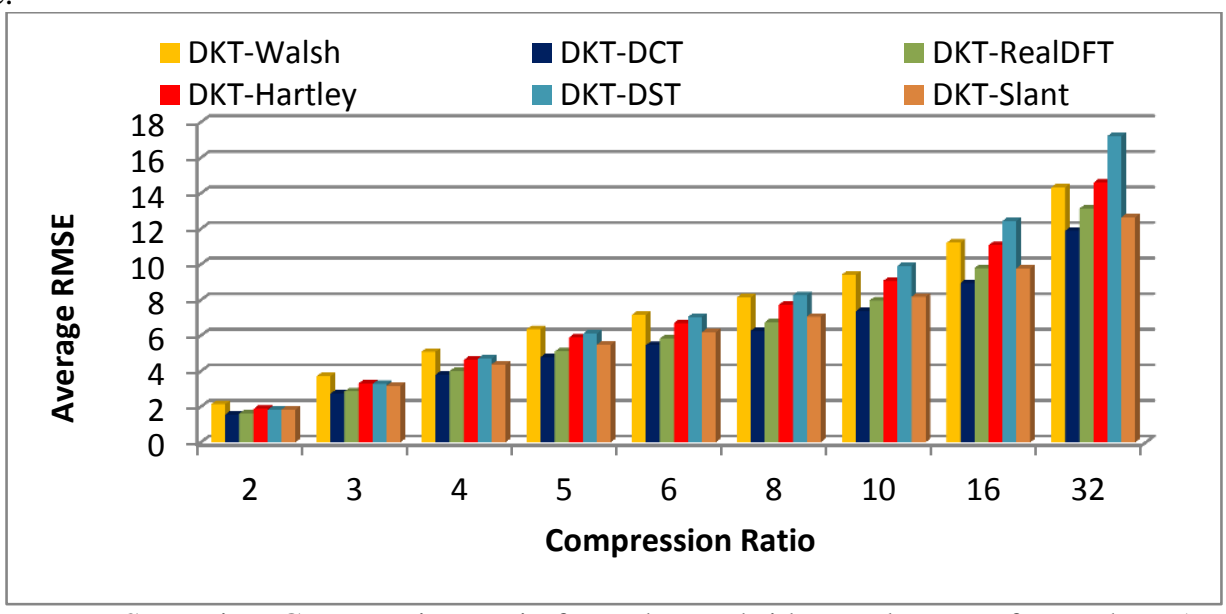

Fig.5Average RMSE against Compression Ratio for Kekre Hybrid Wavelet Transform where 16x16 Kekre Transform acting as a base transform and different local component transforms of size 16x16

Further, as shown in Fig. 6, component size is changed to 32x32 for Kekre transform and 8x8 for second component. DKT-DCT gives lower error followed by DKT-Slant hybrid transform.

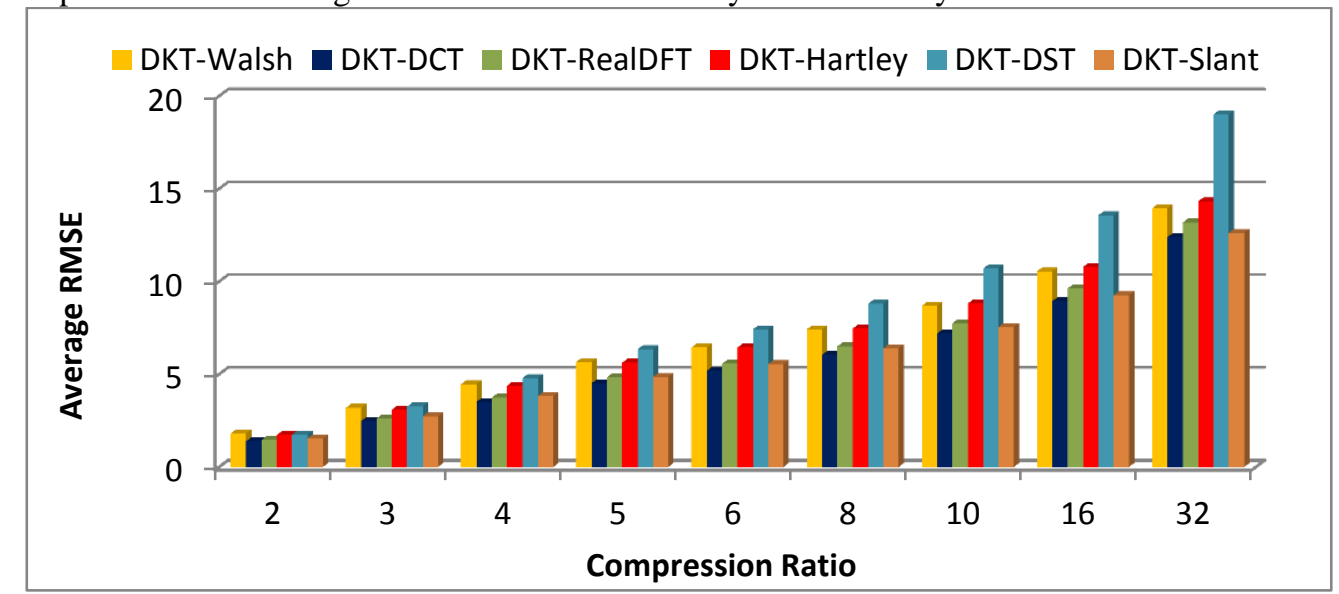

Fig.6Average RMSE against Compression Ratio for Kekre Hybrid Wavelet Transform where 32x32 Kekre Transform acting as a base transform and different local component transforms of size $8 \times 8$ 
Next size variation done in component transforms is $64 \times 64$ and $4 \times 4$. Results are plotted in Fig. 7. Here RMSE value increases than previous cases. DKT-DCT, DKT-RealDFT and DKT-Slant show nearly equal value of RMSE.

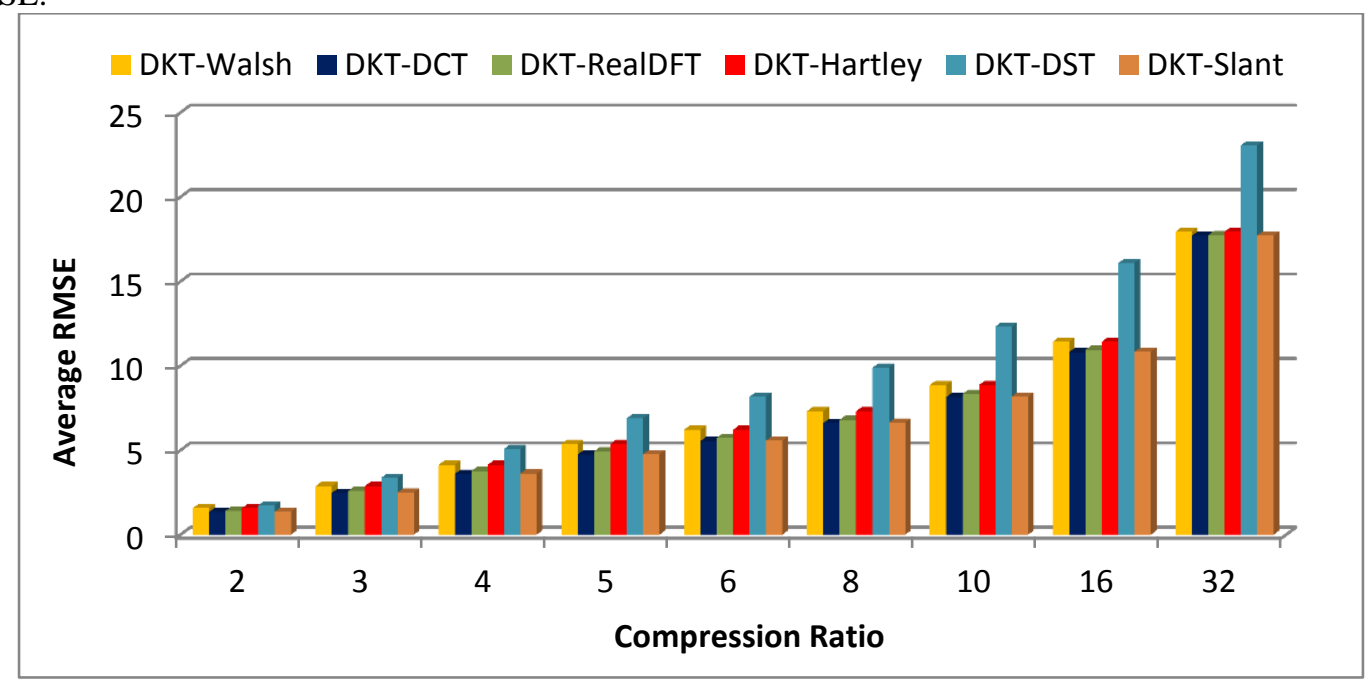

Fig.7Average RMSE against Compression Ratio for Kekre Hybrid Wavelet Transform where 64x64 Kekre

Transform acting as a base transform and different local component transforms of size $4 \times 4$

From above graphs it has been observed that DKT-DCT gives superior performance than other hybrid transforms irrespective of size of component transforms. Resulting compressed images using different hybrid transforms at different compression ratios are shown in Fig.8 with their respective RMSE values. In DKT-DST blocking effect is more prominent than other hybrid transforms and it is observed at compression ratio 32.

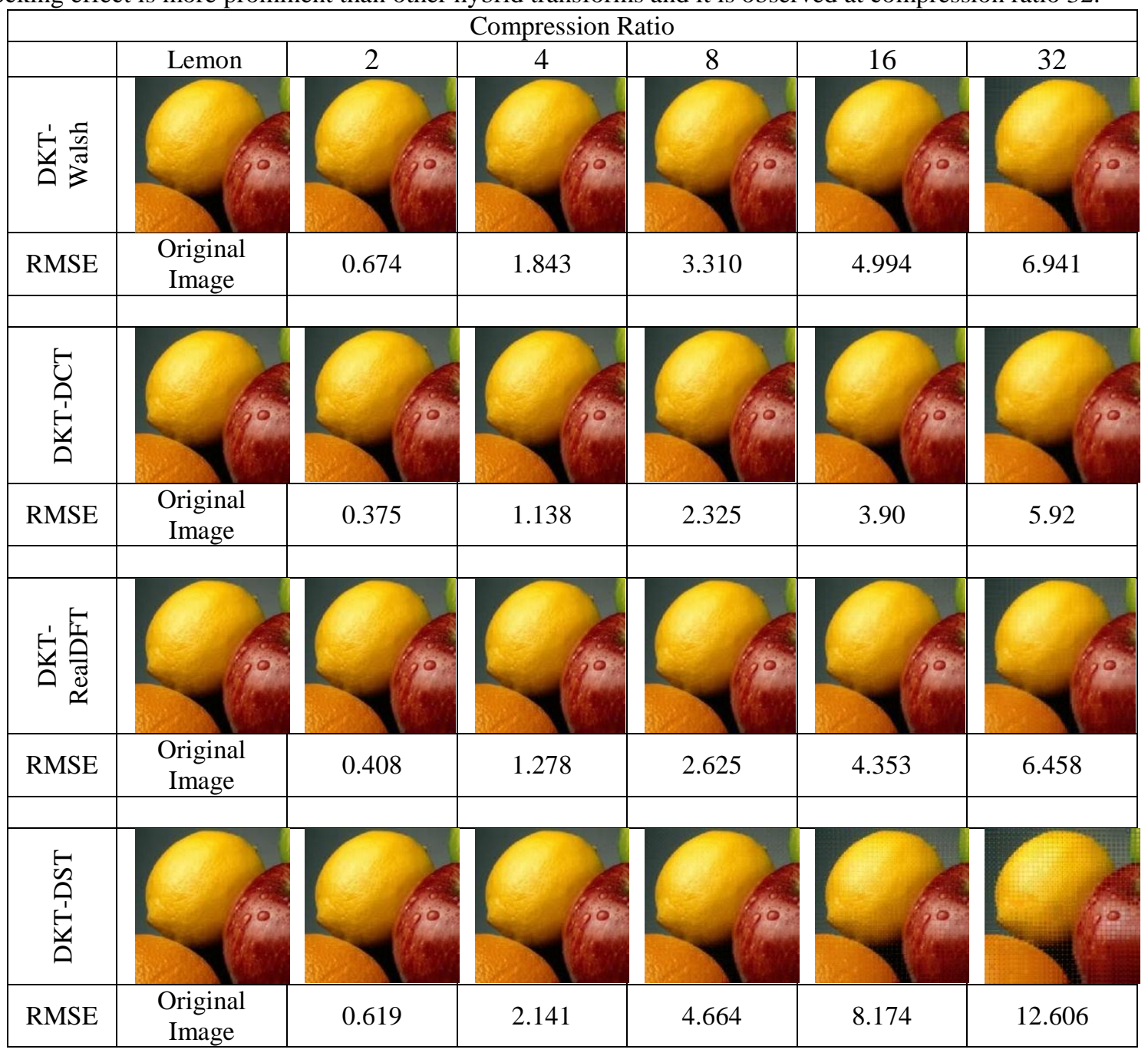




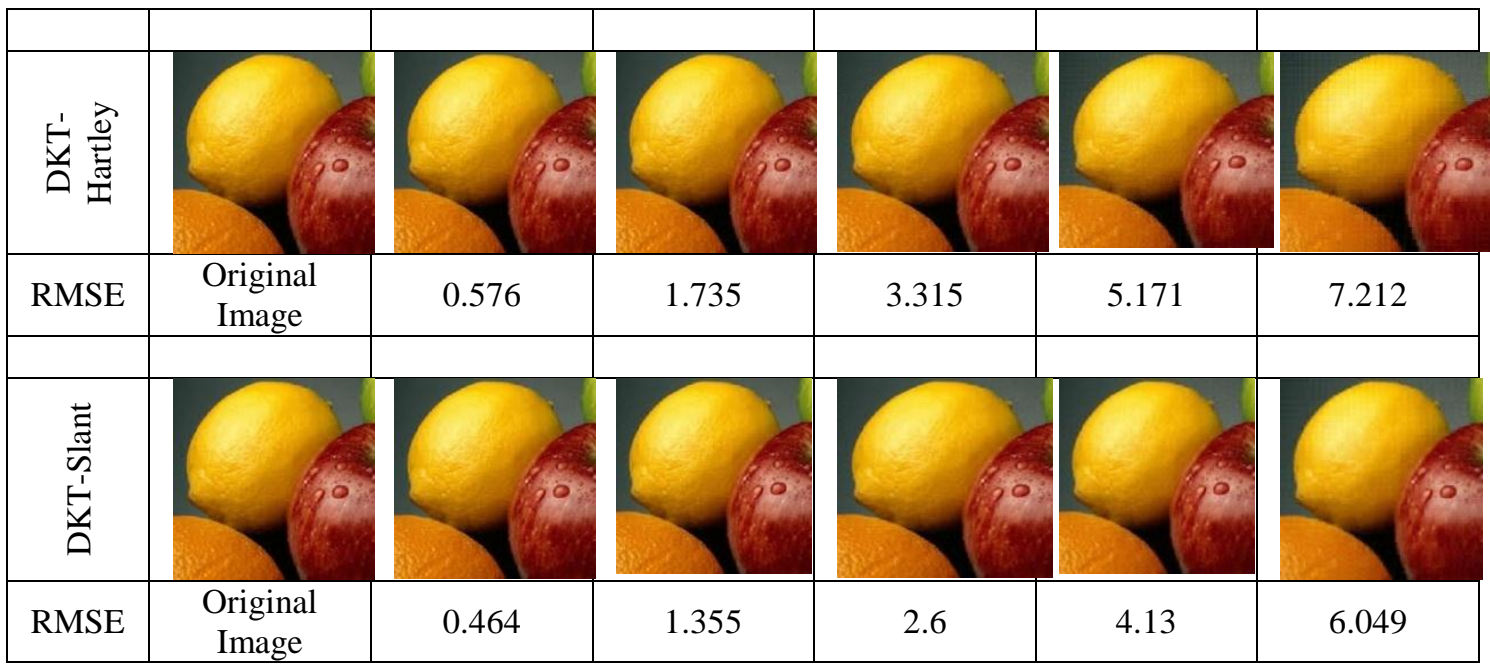

Fig.8 Compressed images obtained using different hybrid transforms at various compression ratios with their respective RMSE values

Fig. 9 shows average RMSE at different bit rates. Less number of bits used to represent the image pixels indicates higher compression. All combinations give acceptable image quality of compressed images at lowest bit rate 0.25 bpp except DKT-DST.

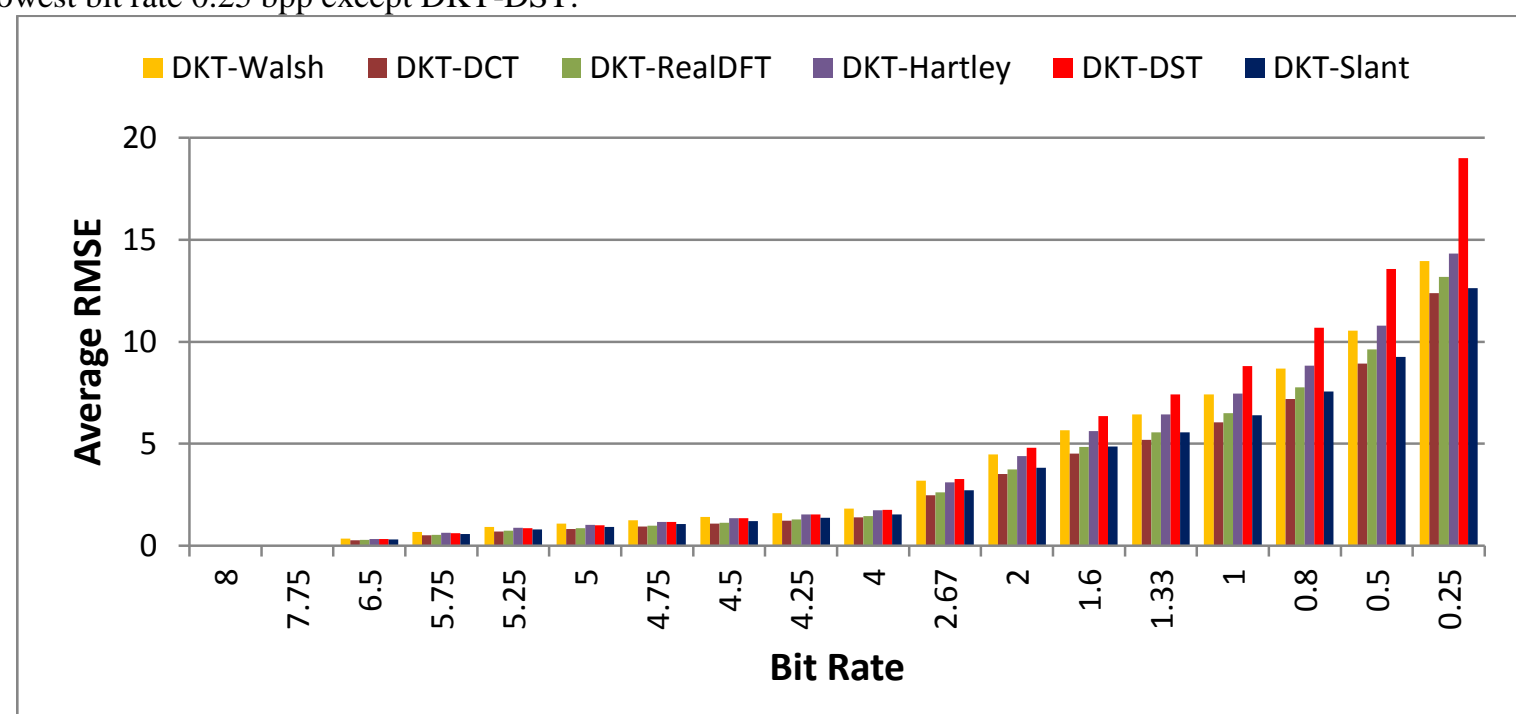

Fig.9 Performance of different hybrid transform in terms of RMSE at different bit rates

Comparison of RMSE values obtained by DKT-DCT having size MNxMNusing various component sizes is tabulated in Table 1.

TABLE 1: RMSE values obtained by DKT-DCT hybrid transform at different compression ratios using variation in sizes of component orthogonal transforms

\begin{tabular}{|c|c|c|c|c|}
\hline \multirow{2}{*}{$\begin{array}{c}\text { Compression } \\
\text { Ratio }\end{array}$} & \multicolumn{4}{|c|}{ Avg. RMSE using DKT of size ' $m$ ', DCT of size ' $n$ ' } \\
\hline & $m=8, n=32$ & $m=16, n=16$ & $m=32, n=8$ & $m=64, n=4$ \\
\hline 2 & 1.7736 & 1.5654 & 1.3850 & 1.3463 \\
\hline 3 & 3.0379 & 2.7324 & 2.4764 & 2.4757 \\
\hline 4 & 4.1601 & 3.7986 & 3.5075 & 3.5999 \\
\hline 5 & 5.1960 & 4.8038 & 4.5132 & 4.7465 \\
\hline 6 & 5.8628 & 5.4586 & 5.1836 & 5.5443 \\
\hline 8 & 6.6895 & 6.2783 & 6.0375 & 6.6058 \\
\hline 10 & 7.7736 & 7.3639 & 7.1918 & 8.1472 \\
\hline 16 & 9.3435 & 8.9583 & 8.9328 & 10.8028 \\
\hline 32 & 12.1830 & 11.9080 & 12.3893 & 17.7049 \\
\hline
\end{tabular}




\section{Conclusion}

Proposed method of image compression uses hybrid transform which is generated using kronecker product of two orthogonal component transforms. Various pairs of component transforms have been tried and it has been observed that DKT-DCT gives superior results as compared to DKT-Walsh. Other transforms like Real-DFT, Hartley, Slant and Discrete Sine transform are used with Kekre transform. DKT-DCT pair gives minimum RMSE with acceptable image quality than other pairs formed with DKT. Component transforms of different sizes are used to generate hybrid transform of size 256x256. DKT-DCT hybrid transform with component size $32 \times 32$ and $8 \times 8$ respectively gives better performance. At compression ratio 16, RMSE of 8.93 is obtained. Even at compression ratio 32, acceptable image quality is obtained with RMSE 11.90.

\section{References}

[1] Ramanjaneyulu. K, Abdul Rahim.B, FahimuddinShaik, “ Effect of Wavelet Based Image Compression Methods on Enhanced Medical Imagery", International Journal of Advanced Research in Computer Science and Software Engineering (IJARCSSE), Vol. 3, Issue 9, September 2013, pp.113-117.

[2] N. Ahmed, T. Natarajan and K. R. Rao, "Discrete Cosine Transform", IEEE Transaction Computers, C-23, January 1974, pp. 90-93.

[3] H.B.Kekre, Sudeep D. Thepade, Juhi Jain, NamanAgrawal, "Performance Comparison of IRIS Recognition Techniques using Wavelet Pyramids of Walsh, Haar and Kekre Wavelet Transforms", International Journal of Computer Applications (IJCA), Number 2, Article 4, March 2011, http://www.ijcaonline.org/proceedings/icwet/number2/2070-aca386.

[4] H.B.Kekre, Sudeep D. Thepade, AkshayMaloo, "Face Recognition using Texture Features Extracted from Walshlet Pyramid", ACEEE International Journal on Recent Trends in Engineering and Technology (IJRTET), Volume 5, Issue 1,www.searchdl.org/journal/IJRTET,2010.

[5] H. B. Kekre, Dhirendra Mishra, "Image Retrieval using DST and DST Wavelet Sectorization", International Journal of Advanced Computer Science and Applications (IJACSA), Vol. 2, No. 6, 2011, pp. 91-97.

[6] H.B. Kekre,A.Athawale, P.N.Halarnkar, V. K. Banura,"Performance comparison of DCT and Walsh Transform for Steganography", ACM, 2010.

[7] Nadenau, M.J., Reichel, J. and Kunt, M., "Wavelet-Based Color Image Compression: Exploiting the Contrast Sensitivity Function", IEEE transactions on image processing, Vol. 12, No. 1, 2003, PP. 58-70.

[8] BibhuprasadMohanty, Abhishek Singh, SudiptaMahapatra, "A High Performance Modified SPIHT for Scalable Image Compression", International Journal of Image processing (IJIP), Volume 5, Issue 4, 2011, pp. 390-402.

[9] A. Said and W.A. Perlman, "A New, Fast and Efficient Image Codec Based on Set Partitioning in Hierarchical Trees," IEEE Trans. on Circuits and Systems for Video Technology, Vol.6, June 1996, pp. 243-250.

[10] K Nagamani, A.G. Ananth, "Ezw and Spiht Image Compression Techniques for High Resolution Satellite Imageries", International Journal of Advanced Engineering Technology (IJAET), Vol.II, Issue II, April-June, 2011, pp. 82-86.

[11] H.B.Kekre, Tanuja Sarode, Prachi Natu, "Efficient Image Compression TechniqueUsing Full, Column and Row Transforms on Colour Image ”, International Journal of Advances in Engineering and Technology, Vol.6, Issue 1, March 2013, pp. 88-100.

[12] H. B. Kekre, Tanuja Sarode, Prachi Natu, "Image Compression Using Column, Row and Full Wavelet Transforms Of Walsh, Cosine, Haar, Kekre, Slant and Sine and Their Comparison With Corresponding Orthogonal Transforms", International Journal of Engineering Research and development (IJERD), Vol. 6, Issue 4, March 2013, pp.102-113.

[13] H.B.Kekre, Tanuja Sarode, sudeep Thepade, Sonal Shroff, "Instigation of Orthogonal Wavelet Transforms using Walsh, Cosine, Hartley, KekreTransforms and their use in Image Compression", International Journal of Computer Science and Information Security (IJCSIS), Vol 9, No. 6, pp. 125-133, 2011

[14] H.B.Kekre, TanujaSarode, PrachiNatu, "Image Compression using Real Fourier Transform, It's Wavelet Transform and Hybrid Wavelet with DCT", Accepted in International Journal of Advanced Computer Science and Applications,(IJACSA) Vol. 4, No.5, pp.41-47,2013

[15] Chandan Singh Rawat, SukadevMeher, "A Hybrid Image Compression Scheme using DCT and Fractal Image Compression”, The International Arab Journal of Information Technology, Vol. 10, No. 6, November 2013, pp. 553-562.

[16] MengMeng, MeijuanZong, "A new ZeroTree Structure for Color Image Compression based on DWT and VQ" In proc.Of $2^{\mathrm{ND}}$ International Conference on Information management and Engineering. April 2010.

[17] K. Uma, P.Geethapalanisamy, P. Geethapoornachandran, "Comparison of Image Compression using GA, ACO and PSO techniques", IEEE-International Conference on Recent Trends in Information Technology, ICRTIT 2011, pp. 815-820.

[18] Khalil M., "Image Compression using New Entropy Coder," International Journal of Computer Theory and Engineering (IJCTE), vol. 2, no.1, 2010, pp. 39-42.

[19] SumeghaYadav, Tarun Kumar Rawat, "Transform Based Hybrid Image Compression Techniques in Conjunction with Fractal Image Compression Scheme" International Journal of Advance Research, (IJOAR) Volume 1, Issue 4, April 2013, pp. 25-35.

[20] SaritaKumari, “ A Wavelet Based Approach for Compression of Color Images", International Journal of Modern Education and Computer Science, January2013, pp. 28-35.

[21] Anil Bhagat, BalasahebDeokate, "Improve Image Quality At Low Bit Rate Using Wavelet Based Fractal Image Coder", International Journal of Advanced Research in Electrical, Electronics and Instrumentation Engineering (IJAREEIE), Vol. 2, Issue 8, August 2013, pp3693-3702.

[22] Dr.H.B.Kekre, Dr.Tanuja K. Sarode, Sudeep D. Thepade, '’Inception of Hybrid Wavelet Transform using Two Orthogonal Transforms and It's use for Image Compression”, International Journal of ComputerScience and Information Security, Vol. 9 , No. 6, 2011.

[23] H.B.Kekre, Sudeep D. Thepade, "Image Retrieval using Non-Involutional Orthogonal Kekre's Transform”, International Journal of Multidisciplinary Research and Advances in Engineering (IJMRAE), Ascent Publication House, 2009, Volume 1, No. I, pp. 189203, 2009. Abstract available online at www.ascentjournals.com

[24] H. B. Kekre, TanujaSarode, PrachiNatu, "Study of Increase in Global Components in Hybrid Wavelets on Data Compression", International Journal of Computer Technology (IJCT), Vol. 9, No.2, July 2013, pp. 1028-1039. 